\title{
Local field effect on infrared phonon frequencies of thin dielectric films
}

\author{
Igor Shaganov**a , Tatiana Perova*b, and Alan Moore ${ }^{\mathrm{b}}$ \\ ${ }^{a}$ Vavilov State Optical Institute, 199034, St.-Petersburg, Russia; ${ }^{b}$ Dept. of Electronic \& Electrical \\ Engineering, Trinity College, Ireland
}

\begin{abstract}
A number of thin dielectric films deposited onto aluminated glass substrates and onto the materials transparent in the IR range (silicon, KRS-5 and CsI) were investigated using infrared transmission and reflection-absorption techniques. The application of these techniques to thin dielectric films at normal and oblique incidence of light allows the observation of both the longitudinal and transverse optical phonons. The longitudinal (LO)-transverse (TO) optical phonons splitting is analysed in terms of a dispersive local field effect. It has been shown that the results of LO-TO splitting obtained from dispersive local field effect are in a good agreement with the results obtained from the dynamical theory of crystalline lattices.
\end{abstract}

Keywords: FTIR spectroscopy; dielectric films; $\mathrm{SiO}_{2} ; \mathrm{MgO} ; \mathrm{ZrO}_{2} ; \mathrm{LO}-\mathrm{TO}$ phonon splitting; local field effect

\section{INTRODUCTION}

As is well known, longitudinal optical (LO) - transverse optical (TO) phonon splitting in infrared (IR) spectra of thin crystalline films is caused by long-range Coulomb forces. The consequent shift of frequencies for $\mathrm{LO}\left(\Delta v_{\mathrm{LO}}=v_{\mathrm{LO}}-v_{0}\right)$ and TO $\left(\Delta v_{\mathrm{TO}}=v_{0}-v_{\mathrm{T} O}\right)$ phonon vibrations from the intrinsic vibrational frequency of crystal $v_{0}$ (so called "bare mechanical frequency") are well described in the frame of point-dipole approximation of the lattice dynamics theory $[1,2]$. In accordance with this theory, LO-TO splitting cannot occur in the spectrum of the structurally disordered (and, in particular, amorphous) condensed media. However, there are experimental evidences that this splitting does occur in the spectra of network glasses. The structural properties of these glasses can be described in the frame of continuous random network (CRN) model suggested in Ref. [3]. Nevertheless, there is some controversy concerning the existence of LO-TO splitting in disordered materials. For example, Phillips [4] has shown that the existence of LO-TO splitting is not consistent with the CRN model for glasses. At the same time, the microscopic phenomenological theory developed in Ref. [5] describes the LO-TO splitting as a result of dipole-dipole interactions between macro parts of glass. The calculations of the vibrational density of states for tetrahedral network glasses, performed in Ref. [6] taking into account the short-range interactions only, support the conclusion made in Ref. [4]. This conclusion states that macroscopic polarisation is absent in the case of tetrahedral network glasses and thus the LO-TO splitting cannot be expected. A quite interesting result was obtained in Ref. [7], where LO-TO splitting has been described in terms of Coulomb interactions between nearest-neighbour atoms and considered as a consequence of the boundary conditions. The calculations based on the consideration of the molecular dynamics of the assembler of a triple generated isotropic oscillators have been performed by Pick and Yvenic [8]. They showed that the vibrational density of states has two peaks that correspond to LO and TO modes. It should be noted that the latter model does not account for the peculiarity of the disorder in glasses and eventually is similar to the results of the Sekimoto model [5]. A more detailed consideration was carried out in Ref. [9] where the glass is considered as a set of the local oscillators such as tetrahedrons $\left(\mathrm{SiO}_{1 / 2}\right)_{4}$.

*perovat@tcd.ie; phone 3531 608-3802; fax 3531 677-2442; http://www.mee.tcd.ie; Department of Electronic \& Electrical Engineering, University of Dublin, Trinity College, Dublin 2, Ireland; **ishag@peterlink.ru; Vavilov State Optical Institute, 199034, St.-Petersburg, Russia. 
Authors of Ref. [9] have considered not only the long-range interactions (as was done in Ref. [10]), but also took into account the effect of disorder in the glass. In accordance with this model the disorder in glasses, caused by the deviation of angles in between the bonds of the tetrahedron anions $\mathrm{SiO}_{4}$, leads to the variation of the local oscillators frequencies and to the broadening of the consequent absorption bands. Of particular interest is a conclusion made in Ref. [9] which stated that the long-range interaction produces a uniform effective electric field which acts as an ordering force and overcomes the effect of the disorder in glass to produce well defined LO-TO splitting.

The intention of this work is to analyse the LO-TO splitting using the semi-empirical theory of the dispersive effective (local) field developed in Refs. [11-13] and apply it to a number of different films such as $\mathrm{MgO}, \mathrm{ZrO}_{2}$ and $\mathrm{SiO}_{2}$. This approach is based on the application of the dispersive local field method accounting for the difference in the dispersion of the average macroscopic $\left(\mathrm{E}_{\mathrm{av}}\right)$ and microscopic effective $\left(\mathrm{E}_{\text {eff }}\right)$ electric fields in a medium. The universality of this approach for the investigation of the influence of the intermolecular interactions on the electronic and vibrational spectra of different condensed media such as crystals, liquids, solutions and amorphous solids was demonstrated during the analysis of a large number of experimental and theoretical data (see reviews $[14,18]$ ).

\section{THEORETICAL CONSIDERATIONS}

In accordance with the theory of the resonance interactions, the LO-TO splitting with respect to the frequencies can be described as follows for the isotropic single crystal:

$$
\begin{aligned}
& \Delta v_{T O}=v_{0}-\sqrt{v_{0}^{2}-\frac{N}{3 \pi c^{2}} q_{t}\left|\frac{\partial \mu}{\partial Q}\right|^{2}} \\
& \Delta v_{L O}=\sqrt{v_{0}^{2}+\frac{2 N}{3 \pi c^{2}} q_{l}\left|\frac{\partial \mu}{\partial Q}\right|^{2}}-v_{0}
\end{aligned}
$$

where $v_{0}$ is the intrinsic frequency of the lattice vibrations, also known as the "bare mechanical frequency" or the frequency of the mechanical oscillator vibrations in the absence of an external electromagnetic field. $\mathrm{N}$ is the number of ionic pairs (oscillators) in unit volume, $(\partial \mu / \partial Q)^{2}$ is the square of the matrix element of the transition dipole moment and $q$ is the local field factor, accounting for the influence of the background polarisation on the frequencies $v>v_{0}$. Here,

$$
q_{t}=\frac{\varepsilon_{\infty}+2}{3} \text { and } q_{l}=\frac{\varepsilon_{\infty}+2}{3 \varepsilon_{\infty}}
$$

where $\varepsilon_{\infty}$ is the dielectric susceptibility of the crystal in the visible range.

In the frame of the dispersive local field approach, the relationship between the spectrum of the intrinsic characteristic of condensed medium $(B(v))$ and the spectrum of the dielectric susceptibility function $(\hat{\varepsilon}(v))$ on the frequency of transverse optical vibrations is as following:

$$
\operatorname{Im} \hat{\varepsilon}(v)=\frac{N h}{2 \pi} B(v)\left|\hat{q}_{t}(v)\right|^{2}
$$

where $\hat{\varepsilon}(v)=\varepsilon^{\prime}(v)-i \varepsilon^{\prime \prime}(v)$ is the complex dielectric susceptibility function and 


$$
\hat{q}_{t}(v)=\frac{\hat{E}_{e f f}(v)}{\hat{E}_{a v}(v)}=\frac{\hat{\varepsilon}(v)+2}{3}
$$

is the factor (a tensor in general case) of Lorentz's local field, accounting for the deference between the effective ( $\left.\mathrm{E}_{\mathrm{eff}}\right)$ and average $\left(\mathrm{E}_{\mathrm{av}}\right)$ fields in the vicinity of the frequency of the vibrational transition under consideration. As was shown earlier, expressions (3) and (4) are valid for any condensed medium without restriction [17].

The term $B(v)$ in Eqn. (3) is the so-called Einstein coefficient [19] spectrum, which represent the spectral density of the specific transition probability of the elementary transition. It should be noted that the term $B(v)$ is differs from the spectrum of the imaginary part of the microscopic susceptibility function of the condensed medium introduced in Ref. [15] by only the normalising coefficient. From another point of view, the physical meaning of the $B(v)$ spectrum can be related to some virtual spectrum, which could represent the vibrational spectrum of the condensed medium in the absence of the optical polarisation on the frequencies of the optical transition or at $v>>v_{0}$. In other words, the spectrum $B(v)$ can represent the intrinsic vibrations of media if it can be measured by non optical methods. As an example of this statement, we can refer to the excellent coincidence of the peak frequency of spectrum $B(v)$ of the abnormal plasma absorption of granulated metallic layers with the peak frequency of the loss spectrum obtained from measurement of the fast electron absorption [14,20].

The frequency maximum of the $B(v)$ spectrum is an intrinsic frequency of the elementary optical transition, which is related to the frequency $v_{0}$ in equations (1) and (2). Therefore, the transition from the absorption spectrum of the condensed media, $K(v)=4 \pi k v$, or its dielectric loss spectrum, $\varepsilon^{\prime \prime}(v)=2 n(v) k(v) \quad$ (where $\hat{n}(v)=n(v)-i k(v)$ is the complex refractive index of this medium), to $B(v)$ spectrum allows the determination of the contribution of the resonance dipole-dipole interactions to the general "red shift" observed in spectra during the transition of molecules from a gas state to a condensed state. In other words, this transition allows one to estimate the role of the dielectric effect on the formation of the consequent spectrum of the condensed medium. Thus, we can state that the dynamic shifts, $\Delta v=v_{B(v)}-v_{\varepsilon "}$, by its nature is similar to the analytical shift, $\Delta v_{\mathrm{TO}}$, described by Eqn. (1). Good agreement between these two shifts was obtained by both methods for a large variety of data for crystals as well as for liquids [11-13,16-18]. In particular, spectrum $B(v)$ can be very close to the spectrum of molecules in a vapour phase. However, the spectrum $B(v)$ should never be identified with the spectrum of the isolated molecule, because the difference in between spectra $B(v)$ and $K(v)$ is related only with the part of the full potential of the intermolecular interactions. This part, in turn, is caused by the effect of the optical polarisation of the media, which can be described in the frame of the model of the Lorentz effective field. The aforementioned correction of the measured spectrum $K(v)$ is based on the latter model.

The type of $q(v)$ function in Eq. (3) follows from the Lorentz model, in accordance with which the average (effective) microscopic field represents the sum of microscopic and local Lorentz's fields:

$$
\hat{E}_{e f f}=E_{a v}+\frac{4}{3} \pi \hat{P}
$$

where $\hat{P}$ is the vector of polarisation or the dipole moment of unit volume, and

$$
\hat{P}(v)=\frac{\hat{\varepsilon}(v)-1}{4 \pi} \hat{E}_{a v}(v) .
$$

In the case of the longitudinal vibrations, the local Lorentz field is equal to: $-\frac{8 \pi \hat{P}}{3}[21]$ and consequently the component of the vector of polarisation, $P_{l}$ is:

$$
\hat{P}_{l}=\frac{\hat{\varepsilon}(v)-1}{4 \pi \hat{\varepsilon}(v)} \hat{E}_{a v}(v)
$$


and therefore from Eq. (7) we can find that

$$
\hat{E}_{e f f}^{l}=\hat{E}_{a v}^{l}-\frac{8 \pi \hat{P}(v)}{3 \hat{\varepsilon}(v)}
$$

and

$$
\hat{q}_{l}(v)=\frac{\hat{E}_{e f f}^{l}}{\hat{E}_{a v}^{l}}=\frac{3 \varepsilon(v)}{\hat{\varepsilon}(v)+2} .
$$

Then, by analogy with Eq. (3), the expression for the spectrum of dielectric loss on the frequency of the longitudinal vibrations can be obtained

$$
A_{L O}=\frac{N h}{2 \pi} B(v)\left|q_{l}(v)\right|^{2}
$$

or finally by substituting of $\mathrm{B}(v)$ with equation similar to Eq. (3) we obtain

$$
A_{L O}=\frac{\operatorname{Im} \hat{\varepsilon}(v)}{|\hat{\varepsilon}(v)|^{2}} .
$$

The obtained Eq. (11) is in agreement with modern theories, stating that the absorption on the frequencies of the longitudinal optical phonons must appear in the range of maximum of the imaginary part of the function $1 / \hat{\varepsilon}(v)[21$ 23]. In the following sections we will apply the dispersive local field theory to the experimental data obtained for a number of thin dielectric films which possesses LO-TO phonon splitting.

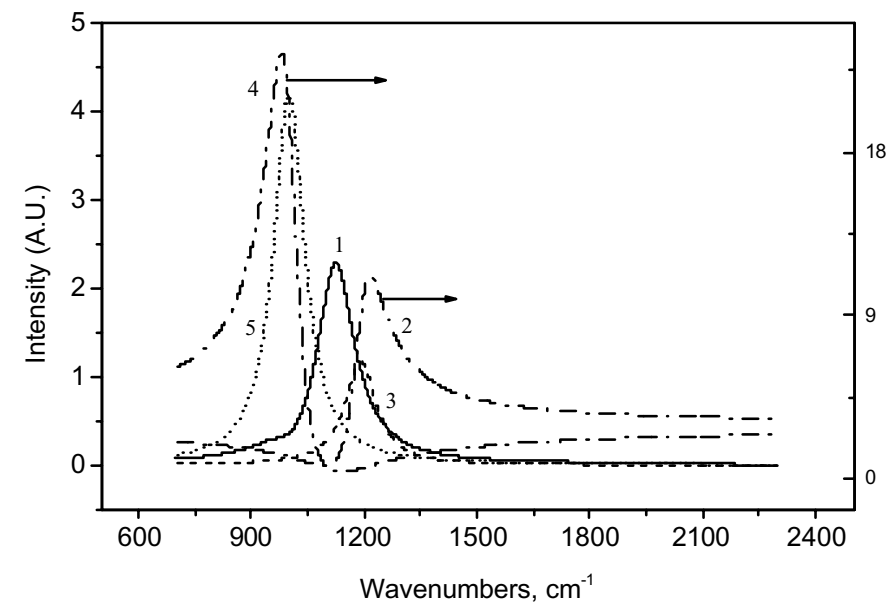

Figure 1. Spectroscopic characteristics of model oscillator in the frequency range of $\mathrm{LO}$ and TO phonon vibrations: 1 - spectrum $B(v), 2-$ spectral dependence of factor $q_{l}^{-1}(v), 3-$ the spectra dependence of the function $\operatorname{Im} \hat{\varepsilon}(v) /\left|\hat{\varepsilon}(v)^{-2}\right|, 4-$ the spectral dependence of the factor $q^{-1}(v), 5$ - the dielectric loss spectrum $\operatorname{Im} \hat{\varepsilon}(v)$.

\section{EXPERIMENTAL}

\subsection{Sample preparation}

Films of $\mathrm{MgO}, \mathrm{ZrO}_{2}$ and $\mathrm{SiO}_{2}$ were deposited using electron-beam evaporation under good vacuum conditions of $\leq 10^{-5}$ Torr and with a deposition rate of $\geq 15 \AA /$ s onto different substrates. Substrates were either metal (aluminium coated glass) or transparent materials such as KRS-5, CsI or Si for IR transmission measurements. $\mathrm{SiO}_{2}$ films were prepared by 
the vacuum evaporation of vitreous quartz pieces in a bell jar evaporator from a heated tantalum crucible. The refractive index $(n)$ and the film thickness $(d)$ were measured using a Gartner L-117 ellipsometer at a wavelength of 632,8 nm.

\subsection{FTIR measurements}

Infrared spectra were measured both in transmission mode at normal incidence of light (to obtain a TO spectrum) and at oblique transmission (at angle of $\sim 60^{\circ}$ ) and in reflection-absorption mode at angle of incidence ranging from $70^{\circ}$ to $80^{\circ}$ (to obtain an LO spectrum). Infrared measurements on the transparent substrates were performed using a Bio-Rad FTS $60 \mathrm{~A}$ spectrometer at normal and oblique incidences of $p$-polarised light in transmission mode. Each spectrum was collected with 64 scans at a resolution of $8 \mathrm{~cm}^{-1}$. Reflection-Absorption Spectra (RAS) were obtained from the aluminium substrates using $p$-polarised light at an angle of incidence of $\sim 75^{\circ}$ using a Perkin-Elmer 283 Infrared Spectrometer equipped with a multi-angle reflection attachment. A calibration spectrum in this case was obtained from a reference aluminium mirror. In the latter case, the optical characteristics (refractive $n(v)$ and absorption $k(v)$ indexes) were determined from the reflection-absorption spectrum, $R(v)$, using simplified equations obtained in Ref. [24] under the assumption that the absorption from the both sides of the vibrational band was zero.
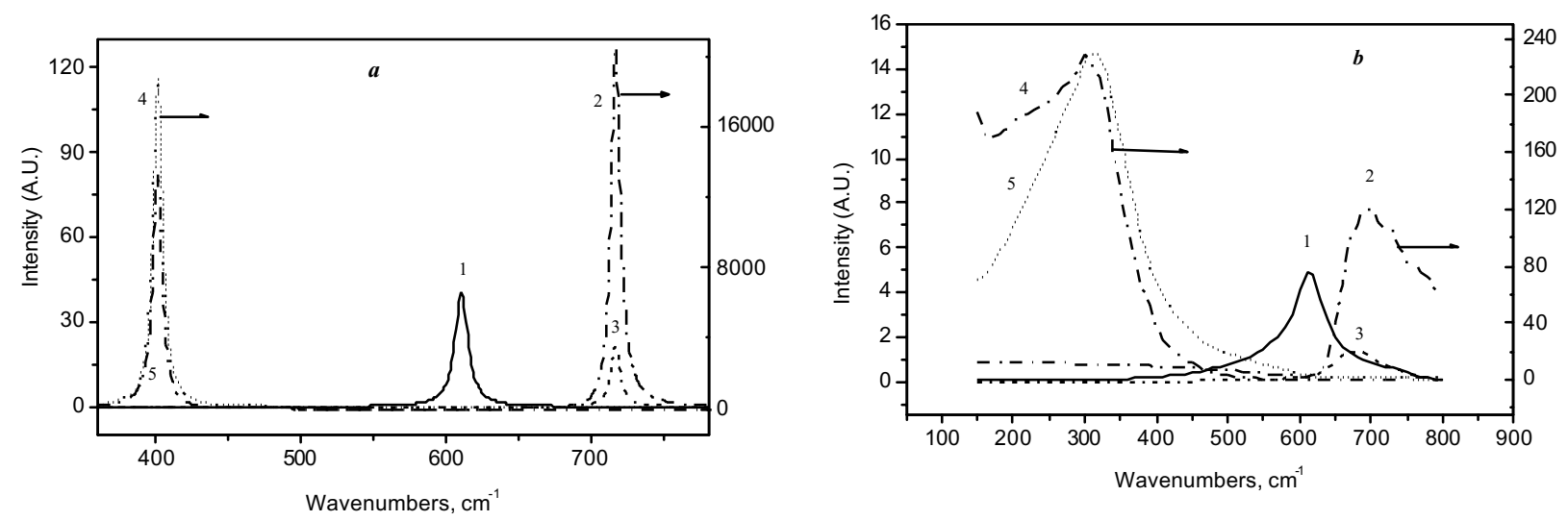

Figure 2. Spectroscopic characteristics of (a) $\mathrm{MgO}$ (crystal) and (b) $\mathrm{ZrO}_{2}($ film) in the frequency range of the lattice vibrations: 1 spectrum $B(v), 2$ - spectral dependence of factor $q_{l}^{-1}(v), 3$ - the spectral dependence of the function $\operatorname{Im} \hat{\varepsilon}(v) /\left|\hat{\varepsilon}(v)^{-2}\right|, 4-$ the spectral dependence of the factor $q^{-1}(v), 5$ - the dielectric loss spectrum $\operatorname{Im} \hat{\varepsilon}(v)$.

\section{RESULTS AND DISCUSSIONS}

The role of the dispersion of $E(v)$ in the formation of the spectral dependence of $\mathrm{A}_{\mathrm{LO}}(\mathrm{v})$ and therefore the spectroscopic characteristics of dielectric media in the range of frequency $v_{\text {LO }}$ may be first illustrated for the model absorption band with a despersive parameters typical for a phonon vibrations of average intensity: $v_{t}=1000 \mathrm{~cm}^{-1}, \rho=0.1, \gamma=0.1$ and $\varepsilon_{\infty}=3$. By knowing these parameters, the refractive $n(v)$ and absorption $k(v)$ indices for this particular transition can be determined from the following equations [25]:

$$
\varepsilon^{\prime}(v)=\varepsilon_{\infty}+\sum 4 \pi \rho v_{j}^{2}\left(v_{j}^{2}-v^{2}\right) /\left[\left(v_{j}^{2}-v^{2}\right)^{2}+\gamma^{2} v_{j}^{2} v^{2}\right]
$$


and

$$
\varepsilon^{\prime \prime}(v)=\sum 4 \pi \rho v_{j}^{2} \gamma v_{j} v /\left[\left(v_{j}^{2}-v^{2}\right)^{2}+\gamma v_{j}^{2} v^{2}\right]
$$

where $\varepsilon^{\prime \prime}(v)=2 n(v) k(v), \varepsilon^{\prime}(v)=n^{2}(v)-k^{2}(v), \varepsilon_{\infty}$ is the high frequency dielectric constant, and $\rho, \gamma$ and $v_{j}$ are the oscillator strength, the damping constant and the frequency of $\mathrm{j}^{\text {th }}$ single harmonic oscillator respectively.

The spectral dependence of the factor $Q(v)=\left|\frac{1}{\hat{q}_{l}(v)}\right|$ which characterises the difference between the spectral density of the average and effective fields, and spectra $\mathrm{B}(v)$ and $\mathrm{A}_{\mathrm{LO}}(v)$, calculated using $n$ and $k$, obtained from the dispersive parameters, are shown in Fig. 1 a. It can clearly be seen that maximum of spectra $A_{L O}$, which characterised the absorption at the frequency $v_{\mathrm{LO}}$, is shifted to the high frequency side with respect to the intrinsic frequency of the vibrational transition $v_{0}$ (or $\left.v_{B(v)}\right)$. This is in accordance with the maximum of the distribution of the spectral density of the longitudinal component $E_{1}(v)$.

At the same time on Fig. $1 b$, similar data for the consequent vibrational band of transverse optical phonons are presented. The analysis of this data shows that the magnitude of LO-TO splitting $v_{L O-T O}=v_{L O}-v_{T O}$ obtained in the frame of the dispersive local field model agrees very well with data obtained from the dynamical theory of crystalline lattices (Eqs. (1) and (2)) which presented in Table 1.

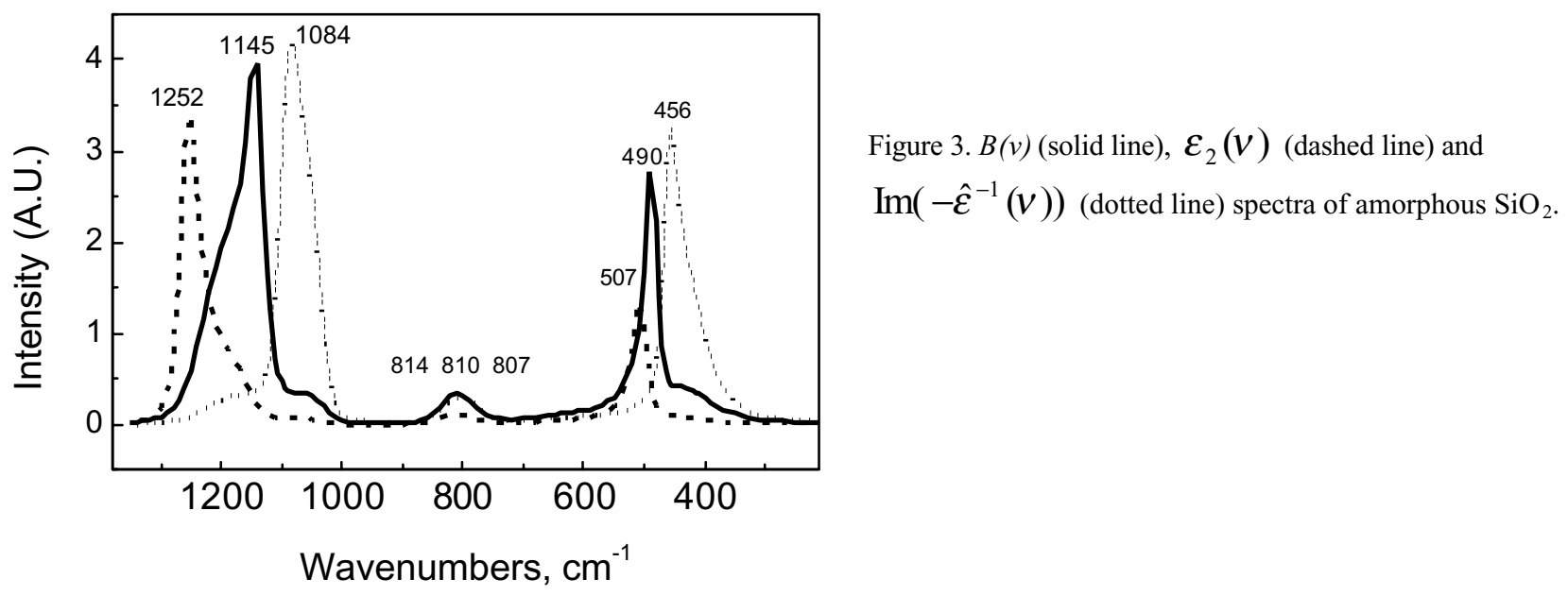

In order to compare the results of the application of the dispersive local field theory to the experiment, we chose a number of thin films such as $\mathrm{ZrO}_{2}, \mathrm{MgO}$ and $\mathrm{SiO}_{2}$. As was shown in Refs. [24,26] for $\mathrm{ZrO}_{2}$ and $\mathrm{MgO}$ and in Ref. [27] for $\mathrm{SiO}_{2}$, the reflection-absorption spectra (RAS) of thin films for these oxides in the range of LO vibrations are reasonably described using the optical constants $(n(v)$ and $k(v))$ of their bulk counterparts. The results of the calculations using Eqns. (3), (4), (9) and (10) in the range of $\mathrm{LO}$ and $\mathrm{TO}$ vibrational modes for $\mathrm{SiO}_{2}, \mathrm{ZrO}_{2}$ and $\mathrm{MgO}$ are presented in Figs. 2 and 3. As one can see from these figures, the peak position $v_{\mathrm{LO}}$ of spectra $A_{L O}(v)$, characterising the absorption at the frequencies of longitudinal phonons, is shifted to the higher frequency side with respect to the intrinsic ("bare") frequency $v_{0}$. The magnitude of this shift is in accordance with maximum of spectral density distribution of the effective field, determined by factor $\hat{q}_{l}(v)$. Similar data for absorption spectra in the range of the transverse optical phonon are also presented in the same plot (see curves 4 and 5). The results of the frequency shifts due to the 
resonance dipole-dipole interactions obtained by the dispersive local field method $\left(\Delta v_{L O}^{r e s}=v_{L O}-v_{B(v)}\right.$ and $\left.\Delta v_{L O}^{r e s}=v_{L O}-v_{B(v)}\right)$ and using Eqns. (1) and (2) are shown in Table 1. As can be seen from Table 1, the resonance shifts obtained with two different theoretical approaches are in a good agreement. Moreover, the reality of the obtained results is confirmed by RAS experimental data for thin films $\mathrm{SiO}_{2}, \mathrm{ZrO}_{2}$ and $\mathrm{MgO}$ obtained in this work and also in Refs. $[24,26,27]$. The data listed in Table 1 shows that the peak position of the absorption spectra obtained from thin films $\left(A_{R}(v)=1-R_{P}(v)\right)$ are very close to the frequency maximum of the bulk optical phonons, $v_{L O}$, obtained in this work using dispersive field effect method. We must note that the agreement between theoretical and experimental data depends strongly on the optical constants $(n(v)$ and $k(v))$ used for the calculation. In particular, if the optical constants of the film were taken from its crystalline counterpart (as in the case of $\mathrm{SiO}_{2}$ ) then the agreement between theoretical and experimental data will depend on the structure (and thickness) of the film under consideration. For example, Fig. 4 shows the $\mathrm{LO}$ absorption obtained for a thick $(\sim 1 \mu \mathrm{m}) \mathrm{SiO}_{2}$ amorphous film (dotted line) and for thin polysilicon film. One can see that the position of LO phonon for polisilicon film $\left(1252 \mathrm{~cm}^{-1}\right)$ is much closer to the calculated one $\left(1258 \mathrm{~cm}^{-1}\right)$, based on optical constants for an $\alpha$-quartz crystal, rather than for an amorphous $\mathrm{SiO}_{2}$ film. This means that the correct optical constants must be used when one performs the calculations.

Table 1 The contribution of resonance dipole-dipole interactions to the frequency of longitudinal optical vibrations for $\mathrm{SiO}_{2}, \mathrm{ZrO}_{2}$, $\mathrm{MgO}$ and model oscillator.

\begin{tabular}{|c|c|c|c|c|c|c|c|}
\hline \multirow[b]{3}{*}{ Sample } & \multicolumn{6}{|c|}{ Crystal } & \multirow{3}{*}{$\begin{array}{l}\text { Film } \\
v_{A_{R}}, \\
\mathrm{~cm}^{-1}\end{array}$} \\
\hline & \multirow[b]{2}{*}{$\varepsilon_{\infty}$} & \multirow{2}{*}{$\begin{array}{c}(\partial \mu / \partial q)^{2} \\
\mathrm{~cm}^{3} / \mathrm{s}^{2}\end{array}$} & \multicolumn{3}{|c|}{ DEF method } & \multirow{2}{*}{$\begin{array}{l}\text { Calc. using } \\
\text { Eq. (2), } \\
\Delta v_{\text {res }}, \mathrm{cm}^{-1}\end{array}$} & \\
\hline & & & $v_{\mathrm{B}}, \mathrm{cm}^{-1}$ & $v_{\mathrm{LO}}, \mathrm{cm}^{-1}$ & $\Delta v_{\text {res. }} \mathrm{cm}^{-1}$ & & \\
\hline $\begin{array}{l}\text { Model } \\
\text { oscillator }\end{array}$ & 3 & & 1125 & 1190 & 65 & 62 & - \\
\hline $\mathrm{SiO}_{2}$ & 2.126 & & 1143 & 1252 & 109 & 101 & $\begin{array}{c}1234^{*} \\
1258^{* *}\end{array}$ \\
\hline $\mathrm{ZrO}_{2}$ & 4.84 & & 595 & 680 & 85 & 78 & $685 \pm 5$ \\
\hline $\mathrm{MgO}$ & 3 & & 610 & 720 & 110 & 106 & $730 \pm 5$ \\
\hline
\end{tabular}

*The position of $\mathrm{LO}$ mode obtained from oblique infrared transmission of $\mathrm{SiO}_{2}$ amorphous film.

** The position of LO mode obtained from oblique infrared transmission for thin film of poly-silicon.

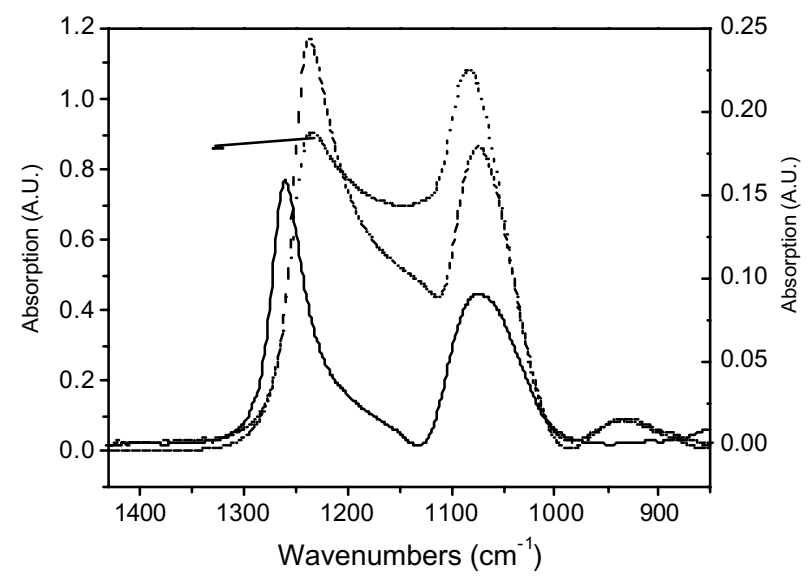

Figure 4. The absorption spectrum of amorphous $\mathrm{SiO}_{2}$ films with different thickness $(1 \mu \mathrm{m}$ - dotted line and $0.2 \mu \mathrm{m}-$ dashed line) and polysilicon) film (solid line measured at angle of incidence of $60^{\circ}$ in oblique transmission mode. 
The data obtained in this work allow us to conclude that the frequency shift observed in the RAS spectra of thin films with respect to the intrinsic frequency of their crystalline counterparts is due only to the resonance dipole-dipole interactions. This shift can be described in the frame of the dynamical theory of crystalline lattices [1,2]. However, it should be noted that the observed correlation in between the peak position of RAS spectra of thin films and $v_{L O}$ of their bulk crystal counterparts takes place only under the condition that $\mathrm{d}<<1 / \mathrm{v}$. For $\mathrm{ZrO}_{2}$, for example, this corresponds to the thickness of film $\mathrm{d}<<50 \mathrm{~nm}$. As is shown in Fig. 5, the increase in thickness for the $\mathrm{ZrO}_{2}$ film leads to the spectrum $A_{R}(v)$ shifting to the high frequency side (from $680 \mathrm{~cm}^{-1}$ to $\sim 780 \mathrm{~cm}^{-1}$ ). The shift of peak position $v$ to $\sim 730-780 \mathrm{~cm}^{-1}$ is probably due to the appearance of the new vibrational mode, since the shoulder at frequency $v_{L O}=680$ $\mathrm{cm}^{-1}$ still exists.

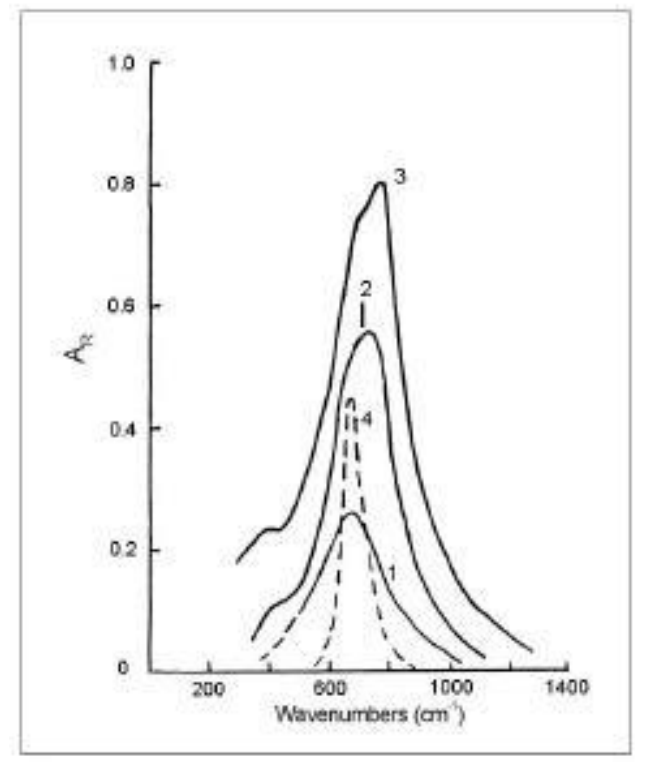

Figure 5 The reflection-absorption spectra of $\mathrm{ZrO}_{2}$ in the frequency range of the LO phonon vibrations calculated from the optical constants of single crystal (1) and measured for the films with different thickness: 2- $\mathrm{d}=50 \mathrm{~nm}, 3-\mathrm{d}=200 \mathrm{~nm}$ and $5-\mathrm{d}=350 \mathrm{~nm}$.

Similar effects were also observed in RAS spectra of thick MgO films [24], where additional absorption bands were found at higher angles of incidence in the range $v>v_{\text {LO }}$. We believe that these additional bands can be related to the excitation of surface polaritons [28] in thick films. The fact that in the RAS spectrum of thin films the only absorption band which shows up belongs to the bulk lattice vibrations, is probably due to the destruction of surface polaritons in vicinity of the metallic surface [23]. It should be noted that although the surface states do not have analogy in the elasticity theory, the spectrum of the surface vibrations are linked to the parameters of the corresponding bulk modes [28] and thus to the intrinsic spectroscopic properties of crystals. Establishing these interactions on the basis of the semi-empirical approach is an important aspect to the manifestation of dipole-dipole interactions in the RAS spectra of metal-dielectric structures and in the investigation of structural and chemical peculiarities of thin dielectric films.

\section{CONCLUSION}

Application of the dispersive local field effect to the analysis of the dielectric loss functions of thin dielectric films at frequencies of the transverse optical and longitudinal optical phonons allows the frequency and oscillator strength of the elementary oscillator, in the absence of resonant dipole-dipole interactions, to be obtained. The frequency of this elementary oscillator $\left(v_{\mathrm{B}(v)}\right)$ coincides with the bare mechanical frequency, $v_{\mathrm{I}}$, introduced in some models using the lattice dynamics theory. The shifts of $\mathrm{LO}\left(v_{\mathrm{LO}}-v_{\mathrm{B}(v)}\right)$ and $\mathrm{TO}\left(v_{\mathrm{B}(v)}-v_{\mathrm{TO}}\right)$ phonons from the maximum of the $B(v)$ 
spectrum, $v_{\mathrm{B}(v)}$, are in a good agreement with these values obtained from the dynamical theory of crystalline lattices. This shows that the calculation of the dispersion of the local field intensity, when applied to the investigation of the resonance interactions in the electronic and vibrational spectra of condensed media [11-14,17], can also be used to describe longitudinal-transverse phonon splitting in glasses.

It should be emphasised that the continual Lorentz model for isotropic media is applicable to liquids as well as to crystals. In accordance with this, we may assume that the dispersive local field approach has a universal character and does not depend on the structure of a condensed media. This can also explain the existence of the LO-TO splitting in thin amorphous dielectric layers. This conclusion is in agreement with results of Ref. [29] where the appearance of the LO component of the IR spectra was explained on the basis of the consideration of the boundary conditions as a result of the space restriction of the condensed media in the direction of the longitudinal vibrations. Therefore, there are a grounds for the assumption that the absorption on the $v_{\mathrm{LO}}$ frequency may be found in liquids also. This work is in progress.

\section{ACKNOWLEDGMENTS}

The authors wish to thank Enterprise Ireland for financial support of this work through the Basic Research Grant Scheme SC/2001/209.

\section{REFERENCES}

1. M. Born and K. Huang, Dynamical theory of crystalline lattices, Oxford, 1954.

2. R. Bonneville, "Local-field factors in cubic crystals", Phys. Rev. 21, pp. 368-376, 1980.

3. F.L. Galeener and G. Lukovsky, Phys.Rev.Lett., 37, pp.1474 (1976).

4. J.C. Phillips, Solid State Physics, vol. 37, p.115, Academic Press, New York, 1982.

5. K. Sekimoto, T. Matsubara, "TO-LO splitting of glassy dielectrics”, Phys. Rev. B26, pp. 3411-3416, 1982.

6. R.J. Bell, A. Carnevale, C.R. Kurkjiaan and G.E. Peterson, J. Non-Cryst. Solids, 35/36, pp. 1185- (1980).

7. A. Lehmann, L. Schulmann, and K. Hubner, "Optical phonons in amorphous silicon oxides. I. Calculation of the density of states and interpretation of LO-TO splittings of amorphous $\mathrm{SiO}_{2}$ ”, Phys.Stat.Sol (b) 117, pp.689-698, 1983.

8. R.M. Pick, M. Yvenic, in Lattice Dynamics, Ed. M. Balkansky, p. 459, Flammarion, Paris, 1978.

9. M.C. Payne and J.C. Inkson, "Longitudinal-optic-transverse-optic vibrational mode splitting in tetrahedral network glasses", J. Non-Cryst. Solids 68, pp.351-360, 1984.

10. P.N. Sen and M.F. Thorpe, Phys. Rev. B15, pp.4030- (1977).

11. N.G. Backshiev, Spectroscopy of intermolecular interactions, Nauka, Leningrad, 1972. (In Russian).

12. N.G. Bakhshiev, O.P. Girin and V.S. Libov, Sov. Opt. \& Spectr., 14, 476, 1963.

13. N.G. Bakhshiev, O.P. Girin and V.S. Libov, Sov. Opt. \& Spectr., 14, 634; 745; 1963.

14. I.I. Shaganov, "Manifestation of local field effects in the properties of optical materials and coatings", Sov. J, Opt. Technol., 59, pp. 1-11, 1992.

15. A.A. Clifford,B. Crawford, "The relation of Optical Constants to Molecular Parameters", J.Phys.Chem. 70, pp. 1536-1543, 1966.

16. T.S.Perova, I.I.Shaganov, J.K.Vij, O.F. Nielsen and P.A. Perov, "Internal field correction of the far-infrared spectra of acetonitrile at different temperatures", Asia Chem. Lett. 4, pp.199-204, 2000.

17. I.I. Shaganov, V.S. Libov, Sov Solid State Phys. 17, pp.1749-1752,1975.

18. V.S. Libov, "Applications of the local field method to the spectroscopic manifestations of resonance interactions in a condensed medium", Rus. J. Phys.Chem. 54, pp. 475-481, 1980.

19. V. Haitler, Quantum theory of emission, Mir, Moscow, 1956.

20. I.I. Shaganov, "Investigation of the effect of resonance interactions on the spectroscopic characteristics of anomalous plasma absorption bands of thin metal films", Rus. Opt. Spectrosc., 49, pp. 181-184, 1980.

21. N.W. Ashcroft and N.D. Mermin, Solid State Physics, Saunders College Publishing, Troy, Missouri, 1976.

22. D.W.Berreman, "Infrared absorption bands at polar longitudinal optic mode frequencies in cubic crystal films", $J$. Phys. Chem. Solids, Supplement 1, pp.397-402,1965. 
23. V.M. Agranovich, A.G. Mal'shikov and M.A. Mekhtiev, "The surface excitons in dielectric on the interface with metal", Rus. Solid State Physics, 14, pp. 849-857 1972.

24. O.P. Konovalova, O.Yu. Rysakova and I.I. Shaganov, "The determination of the optical constants thin films evaporated onto the metal in vacuum from IR reflection", Sov.J.Opt.Technol. 55, pp. 402-404, 1988.

25. A.M. Efimov, Optical Constants of Inorganic Glasses, CRC Press, Florida, 1995, pp.164-166.

26. I.I. Shaganov, E.I. Fadeeva, E.N. Eliseev and O.P. Ankudinova, "Infrared investigation of structural features of thin films of $\mathrm{ZrO}_{2}$ ", Rus. J. Applied Spectrosc. (in Russian), 39, pp.253-258, 1983.

27. I.I. Shaganov, T.S. Perova, R.A. Moore and K. Berwick, Spectroscopic characterisation of $\mathrm{SiO}$ and $\mathrm{SiO}_{2}$ solid films: Assignment and local field influence, J.Material Science: Materials in Electronics, 12, pp. 351-355, 2001.

28. Surface polaritons, Eds. V.M. Agranovich and M.M. Maradudin, M. Nauka, 1985, p.522.

29. A. Lehmann, "Theory of infrared transmission of thin insulating films", Phys. Stat. Solidi B148, pp. 401-405, 1988. 